\title{
Reconstrucción del sector anterior con resinas compuestas
}

Upper anterior zone restoration with composites

\begin{abstract}
Resumen
Los problemas en el sector anterior son muy comunes en nuestra práctica profesional y cobra vital importancia el realizar una adecuada rehabilitación en estos casos; no podemos hacer una buena rehabilitación si no conocemos las características básicas tanto estéticas como funcionales. Hoy las resinas compuestas son una alternativa válida para la reconstrucción del sector anterior, ya que nos brindan una buena posibilidad conservadora y estética, pero independientemente del material a utilizar debemos basarnos en ciertas reglas o parámetros para realizar una adecuada rehabilitación. En el presente artículo se detallan los criterios básicos a tomar en cuenta para reconstruir el sector anterior así como la técnica al utilizar resinas compuestas.
\end{abstract}

\section{Abstract}

The anterior sector problems are very common in our professional practice and became vital importance to make a suitable rehabilitation in these cases; we can not do a good rehabilitation if we do not know the basic characteristics, both aesthetic and functional. Today the composites are a valid alternative for the restoration of the anterior sector, since they offer to us a conservative and aesthetic possibility, but independently of the material to use we have to based on certain rules or parameters to realize a suitable rehabilitation. In this article the basic criteria to keep in mind are detailed to restore the anterior sector as well as the skills on having used composites.

\section{Introducción}

Conocer las características anatómicas $y$ funcionales de las piezas en el sector anterior es de vital importancia para realizar restauraciones duraderas. No basta con realizar una restauración solamente estética si esta no cumple con los requisitos de función del sistema estomatognático.

Tanto las restauraciones directas como las indirectas tienen ventajas y desventajas, y está en el profesional saber elegir cual procedimiento realizar. ${ }^{1}$

El sector antero superior cumple un papel muy importante en la estética del rostro, ${ }^{2,3}$ por lo que conocer a profundidad sus características por parte del profesional es necesario para realizar una adecuada restauración.

La condición de salud periodontal es muy importante para aumentar las expectativas de éxito en nuestros tratamientos, en una pieza dental con problemas periodontales no debe ser rehabilitada si antes no solucionamos este problema. Un tratamiento restaurador solo estará completo cuando se le eduque al paciente para realizarle tratamientos de mantenimiento y así lograr mantener su salud periodontal. ${ }^{4}$
Los dientes anteriores se inclinan hacia distal en sentido del ápice radicular 6 grados aproximadamente. Este efecto de distalización de las piezas en el sector anterosuperior debemos de reproducirlo cuando realicemos nuestras restauraciones. ${ }^{5}$

El contorno gingival de los incisivos laterales está situado más coronalmente en relación con los centrales y caninos. ${ }^{6} \mathrm{Se}$ considera estéticamente aceptable cuando el contorno de gingival del incisivo lateral se encuentra ubicado a la misma altura del incisivo central y del canino.

El nivel del contacto interdental va aumentando en altura hacia distal; es decir que el punto de contacto entre los incisivos centrales está ubicado más hacia incisal, el punto de contacto entre el incisivo central y el incisivo lateral es más alto y el punto de contacto entre el incisivo lateral y el canino está ubicado más alto que los dos anteriores. ${ }^{1}$

En un paciente joven el borde incisal de los incisivos centrales superiores es 2 a $3 \mathrm{~mm}$ más largo que el labio superior cuando este está en reposo. En un paciente con edad avanzada este borde se reduce y el borde incisal coincide con el borde inferior del labio superior. ${ }^{7}$ Se considera estéticamente aceptable cuando el borde incisal de los dientes sigue el contorno del borde superior del labio inferior. ${ }^{5}$

\section{César Lamas Lara ${ }^{1}$ Giselle Angulo de la Vega ${ }^{2}$}

1 CD, Docente del Área de Operatoria Dental y Endodoncia de la Facultad de Odontología de la UNMSM.

2 CD, Alumna de la Especialidad de Rehabilitación Oral de la Facultad de Odontología de la UNMSM.

\section{Correspondencia:}

CD César Lamas Lara

Dirección: Facultad de Odontología de la UNMSM.

Av. Germán Amézaga s/n. Lima 1. Perú

Teléfono: 999-491403

E-mail: cesar2579@hotmail.com

Palabras clave: resinas compuestas, normas estéticas, aplicaciones clínicas.

Keywords: Composites, aesthetic procedure, clinical application.

La Proporción Áurea no sólo simboliza belleza en un nivel primario sino que también es la clave de gran parte de la belleza normal.

La relación entre ancho y alto de los dientes en conjunto tienen un efecto significativo en la apariencia visual del conjunto. ${ }^{8}$

Se dice que el diámetro mesiodistal del incisivo superior está en divina proporción con el diámetro mesiodistal del incisivo inferior y que las piezas dentarias en el sector antero superior también siguen la proporción divina.

Las posibilidades para el uso de las resinas compuestas han aumentado debido a sus excelentes características como: resistencia mecánica, estabilidad de color, adaptación marginal, características que están promoviendo cada vez más su uso. ${ }^{?}$

Cuando se utiliza una fuente de activación con intensidad menor a $300 \mathrm{~mW} /$ $\mathrm{cm}$ puede causar disminución de la fuerza de unión de los adhesivos dentinarios, con riesgo de daño pulpar, disminución de la estabilidad de color y disminución de la resistencia al desgaste. ${ }^{10}$

Antes de realizar cualquier procedimiento restaurador debemos considerar los posibles problemas estéticos y de acuerdo a la situación elegir el tipo de material idóneo para cada caso. ${ }^{11}$ 


\section{Caso clínico}

Paciente sexo femenino de 20 ańos de edad que acude a la Clínica de la Facultad de Odontología de la Universidad Nacional Mayor de San Marcos presentando lesiones cariosas en el sector anterior preocupada por su problema estético.

$\mathrm{Al}$ examinar el sector antero superior se encontraron lesiones cariosas a nivel de las piezas $1.2,1.1,2.1$ y 2.2 . Fig. 1 .

\section{Diagnóstico}

Del estado de salud general: Paciente con aparente buen estado de salud general, sin riesgo sistémico al tratamiento estomatológico.

Del estado de salud estomatológico: Paciente con presencia de lesiones cariosas en piezas 1.2, 2.1, 2.2 y 1.1 además presenta giroversiones de las piezas 1.2 y 2.2 y vestibularizaciones de las piezas 1.1 y 2.1 .

\section{Plan de tratamiento}

Se realizó las pruebas de sensibilidad pulpar para determinar la vitalidad de las piezas sobre todo en las piezas $1.1 \mathrm{y}$ 2.1 que presentaban lesiones que comprometían más estructura. Se realizó la toma de modelos de estudio y se procedió a llevar a cabo el encerado de nuestro caso con la finalidad de confeccionar una guía de silicona (fig. 2) para realizar una reconstrucción con resina compuesta de las piezas dentarias comprometidas.

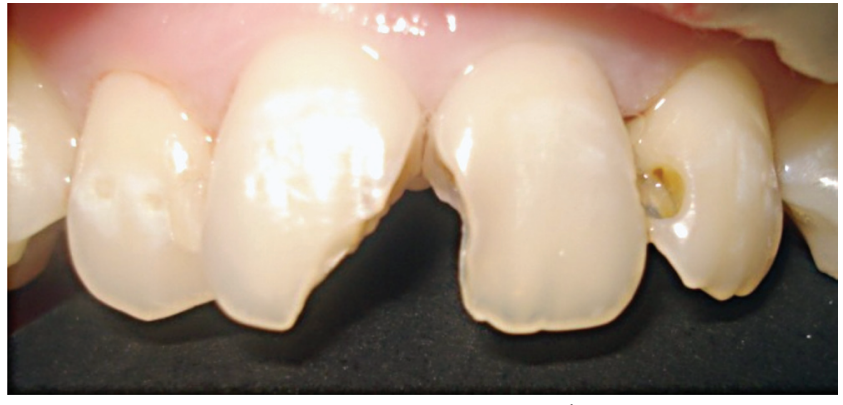

Fig. 1. Situación inicial.

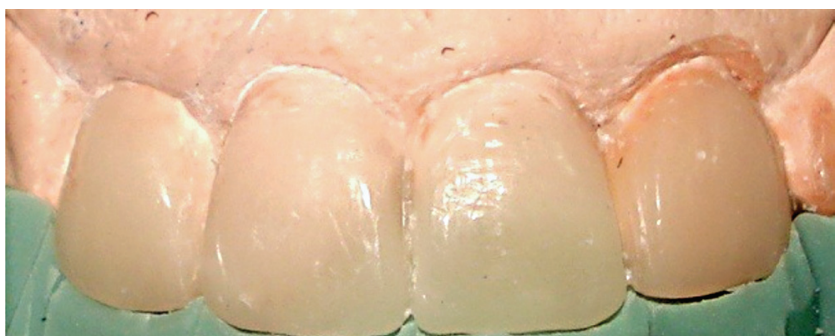

Fig. 3. Biselado de las superficies dentarias.

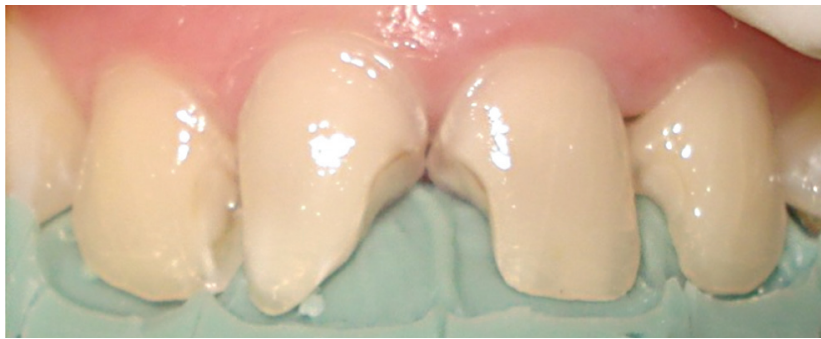

Fig. 5. Aplicación de adhesivo y fotocurado por $20 \mathrm{seg}$. Ubicación de la guía de silicona.

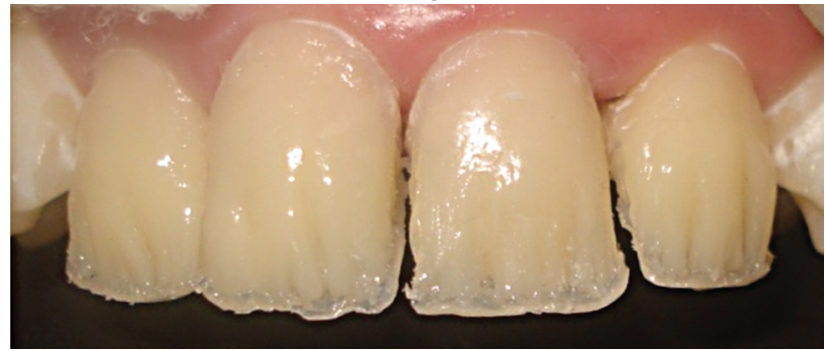

Fig 7. Aplicación de una resina de cuerpo color A2.

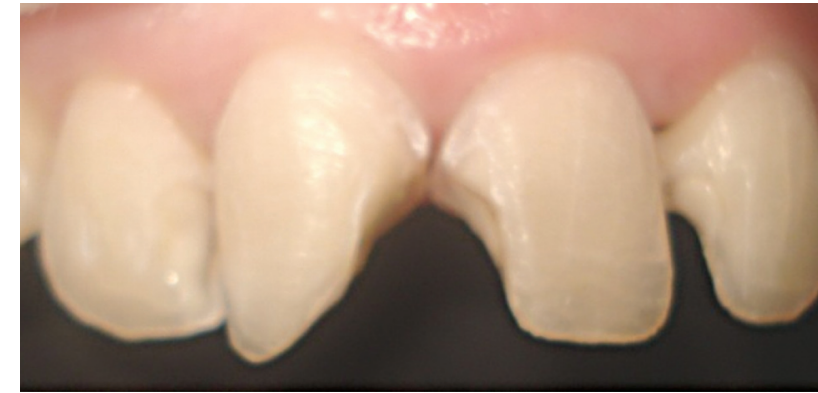

Fig. 2. Encerado y confección de la guía de silicona.

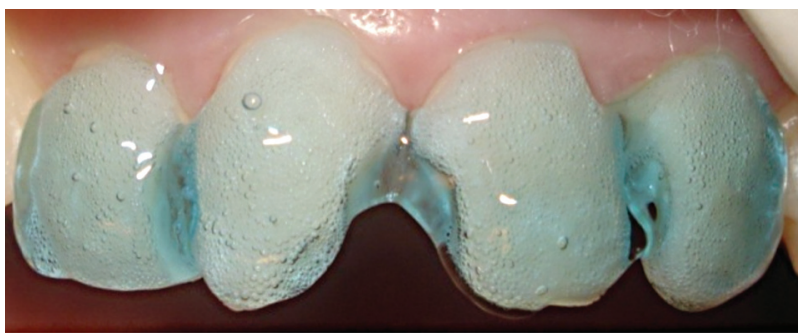

Fig. 4. Grabado ácido por 15 seg., lavado y secado.

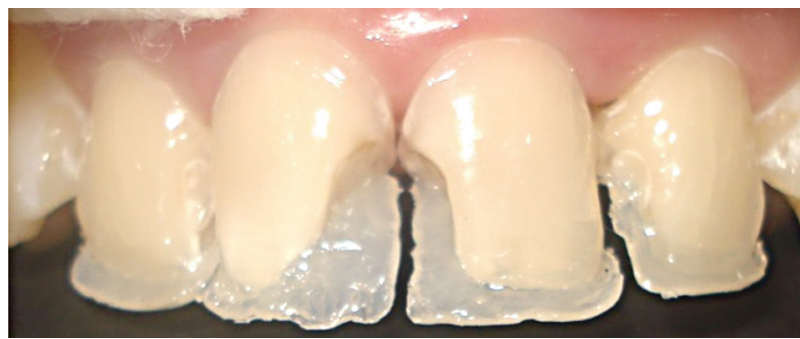

Fig 6. Aplicación de una resina translúcida en palatino.

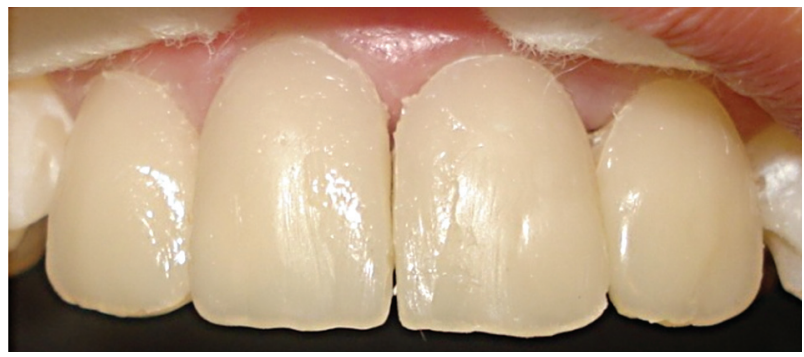

Fig 8. Aplicación de una resina de esmalte color A1.

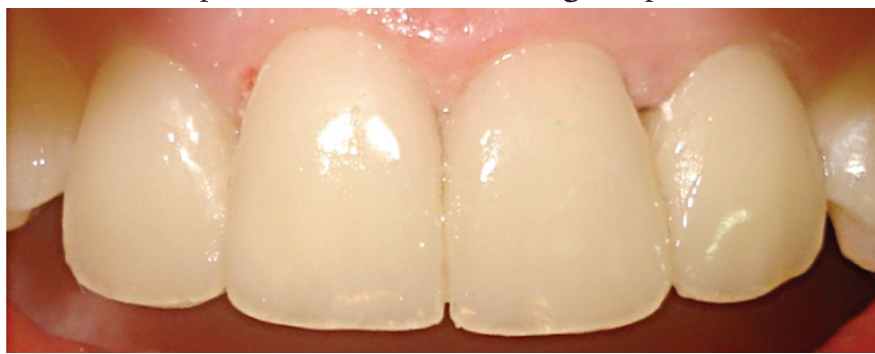

Fig 9. Restauraciones terminadas. 


\section{Procedimiento clínico para la reconstrucción del sector anterior con resinas compuestas}

Se realizó la remoción de las lesiones cariosas y la protección pulpar respectiva con ionómero de vidrio en las partes más profundas y las preparaciones respectivas regularizando y biselando las superficies. (Fig. 3). Se seleccionó el color basándose en la escala Vita, eligiendo para dentina A2, para el esmalte palatino un color traslúcido y para vestibular A1. Se eligió una resina microhíbrida, debido a sus propiedades mecánicas y estéticas. Se procedió al aislamiento del campo operatorio utilizando separadores de labio y lengua, rollos de algodón y eyectores de saliva. Efectuamos el grabado ácido de las superficies preparadas con ácido orto fosfórico al $37 \%$ por 15 seg. (Fig. 4), lavado, secado y la aplicación de un adhesivo de quinta generación y su fotopolimerización por 20 seg. (Fig. 5).

Se utilizó una resina traslúcida para realizar el esmalte palatino utilizando la guía de silicona para su aplicación (Fig. 6); se aplicó una resina de dentina de color A2. (Fig. 7) y para el esmalte vestibular se aplicó una resina para esmalte color A1. (Fig. 8). Cada incremento de resina fue fotopolimerizado por $20 \mathrm{seg}$.

Posteriormente se realizó el acabado y pulido de las restauraciones apreciándose restauraciones estéticamente aceptables. (Fig. 9).

\section{Discusión}

Las restauraciones en el sector anterior requieren por parte del profesional conocimientos tanto teóricos, científicos y artísticos, así como habilidad manual con el fin de reproducir los detalles anatómicos. ${ }^{12,13}$

El uso de materiales cerámicos siempre va a ser una muy buena alternativa para estos casos, por sus demostradas bondades; pero las resinas compuestas de uso directo cobran vital importancia ya que nos dan una posibilidad más en la resolución de nuestros tratamientos, al presentar preparaciones más conservadoras, materiales estéticos adecuados, menores tiempos clínicos y resultados previsibles. Aunque las propiedades de resistencia y estabilidad del color sean menores en las resinas en relación con las cerámicas, aun así son satisfactorias. ${ }^{14,15}$

\section{Conclusiones}

La practicidad de las resinas compuestas la convierten en una excelente opción para los problemas en el sector anterior.

El íntimo conocimiento de las formas de las piezas dentarias en el sector anterior así como su relación con las demás piezas nos va a llevar a maximizar nuestros resultados tanto estéticos como funcionales.

No basta con realizar una restauración que visualmente se vea correcta si esta no cumple su función dentro de la arcada dentaria.

El conocimiento profundo de las diferentes propiedades de las resinas compuestas que el profesional va a utilizar, independientemente de las marcas comerciales, va a facilitar la ejecución de las mismas. Cabe destacar que no todas las resinas compuestas se comportan de la misma manera, tanto en sus propiedades físicas y mecánicas como en su manipulación.

\section{Referencias bibliográficas}

1. Baratieri L, et al. Estética, Restauraciones adhesivas directas con resinas compuestas en dientes fracturados livraria santos Editora, 2004, 2da 4, 24.

2. Jenny J et al. Relationship between dental esthetics and attribution of self-confidence. J Dent Res 1990 69:204

3. Jenny J et al. Visibility and prestige of occupations and the importance of dental appearance. J Can Dent Assoc 1986; 12:987-989.

4. Tumenas, I Ishikiriama S.M. Planeamiento estético integrado em periodontal dentística. Sao Paulo,
Artes Médicas, $20^{\circ}$ CIOSP, Cap 13:v.3, 2002.

5. Miyashita E et al. Odontología Estética: El estado del Arte Sao Paulo: Ed. Artes medicas, 508-513, lera ed. 2005

6. Rufenatch CR. Fundamentals of esthetics. Berlin: Quintessence, p. 67-134, 1990

7. Yamamoto M. et al Special discussion Fundamental esthetics: Contouring techniques for metal ceramic restorations. Quintessence Dent Tech 1990-1991; 14: $10-81$.

8. Albers HF, Tooth colored restoratives. Catati: Alto Books, 1985, 7:31-32.

9. Chain MC, Baratieri LN. Restauraciones estéticas con resina compuesta en dientes posteriores. São Paulo: Artes Médicas/EAP-APCD. 1998.

10. Castro F. Avaliação da intensidade de energia luminosa e do calor produzidos por aparelhos fotopolimerizadores. RBO. 2001; v58:3; 204-206.

11. Sabtana U. Restauración Prostodóntica del complejo dentina raíz: Perno Muñón-Colado. Ed. Quintessence books. 1999.

12. Nogueira de Sá MP, Pascotto RC. Faceta direta em resina composta com recurso de uma matriz de acetato-relato de caso clínico. Revista dental press de estética. 2004; $1: 101-111$

13. Bloom DR, Padayachy J N. Smile lifts-a functional and aesthetic perspective. Br Dent J 2006; 200: 199-203.

14. Fahl N. A polychromatic layering approach for solving a complex class IV/ direct veneer/ diastema combination: Part II. Pract Proced Aesthet Dent 2007;19:17-22.

15. Medeiros C, Aesthetics Rehabilitations through direct facets - Report of Cases. Acta Odontológica Venezolana 2009; v47:2; 1-14.

Fecha de recepción: 9 de diciembre 2009

Fecha de aprobación: 5 de enero 2010 